\title{
Abstracts from the winter meeting of the Scottish Otolaryngology Society, Southern General Hospital, Glasgow, Scotland, UK, 25 November 2006
}

\section{Two fingers for salivary surgery?}

N Brownson, N Calder, A Murray

From the Department of Otolaryngology, Crosshouse Hospital, Kilmarnock, Scotland, UK

\section{Objectives}

Most surgeons performing salivary gland surgery are familiar with the concept of using two finger breadths as a measurement below the mandible in order to safely avoid damage to the mandibular branch of the facial nerve. The authors reviewed the literature to look for the basis of this concept, asking the question, 'are two fingers always sufficient as a measurement in salivary gland surgery?' There was noted to be some ethnic variation. Whilst, in the majority of specimens, the nerve was found within $1 \mathrm{~cm}$ of the lower border of the mandible, there were specimens in which this distance was up to $3 \mathrm{~cm}$.

\section{Design}

With this outside measurement of $3 \mathrm{~cm}$ in mind, an anthropometric study was designed, measuring two and three finger breadths and recording surgical glove size in 50 voluntary subjects.

\section{Participants}

Volunteer members of the medical and nursing staff.

\section{Results}

All females participants had a glove size in the range 5.5-7 $(n=25)$. All male participants had a glove size in the range 7-8 $(n=25)$. There was no relationship between gender and finger breadth once glove size was taken into account. The data could therefore be considered as one set, and it was found that glove size was a significant predictor of finger breadth.

\section{Conclusion}

It is only safe to use two finger breadths if the surgeon's glove size is 8 or more. For surgeons with glove size of 5.5 or more, it is safe to use three finger breadths.

\section{What is the value of cancer screening clinics?}

\section{A Isa, A Murray}

From the Department of ENT Head and Neck Surgery, Crosshouse Hospital, Kilmarnock, Scotland, UK

\section{Objective}

The aim of this study was to clarify the effectiveness of hoarse voice clinics in detecting patients with laryngeal cancer in the Ayrshire and Arran area.

\begin{abstract}
Method
Firstly, we collected and analysed data collected prospectively on patients seen at the hoarse voice clinics (run by the second author) from September 2001 to April 2004. Secondly, we analysed the cases of patients diagnosed with laryngeal cancer during this period, from the pathology department's database. Thirdly, we reviewed the case notes of those patients diagnosed with laryngeal cancer during this period who had not been seen at the hoarse voice clinic, to determine their clinic of origin.
\end{abstract}

\section{Results}

Three hundred and eighteen patients were seen in the hoarse voice clinic from September 2001 to April 2004. Of these, seven patients were diagnosed with laryngeal cancer (2 per cent of clinic patients).

A further 54 patients were found to have laryngeal cancer on biopsy during the study period. Case notes for these patients were requested in order to determine which clinics they were seen in. Only 38 patients had complete information in their case notes. Their clinics of origin were as follows: consultant A clinic, five patients; consultant B clinic, three; staff grade clinic, three; emergency ward admissions, two; consultant $\mathrm{C}$ clinic, 11; neck lump clinic, seven; and consultant D clinic, seven.

Of these 38 patients, 29 were hoarse at presentation. However, the other nine patients presented with normal voice but suffered other symptoms (24 per cent).

\section{Discussion}

Although 61 patients were found to have laryngeal cancer during the study period, only 45 cases could be studied due to either lack of case notes or incomplete case note information. We will refer to these 45 patients as the study population.

Only 15 per cent of the study population were diagnosed from the hoarse voice clinic. Although small numbers were diagnosed in general ENT clinics, a significant number originated from the neck lump clinic (15 per cent) and from consultant C's (head and neck) clinic ( 24 per cent). The majority of the study population had a hoarse voice at presentation, although nine patients (20 per cent) had other symptoms.

In conclusion, hoarse voice clinics are not an effective method of detecting laryngeal cancer, due to the following. Firstly, only a small percentage ( 2 per cent) of patients seen at the hoarse voice clinic were diagnosed with laryngeal cancer. Secondly, comparable numbers of laryngeal cancers (24 per cent) were diagnosed from head and neck clinics. And thirdly, 3.2 per cent of patients in the study population had a normal voice.

Although the numbers diagnosed with laryngeal cancer from the hoarse voice clinic could increase with more rigid referral protocols, increased numbers of patients referred to the hoarse voice clinic may impact on waiting 
times, without necessarily increasing the overall percentage detected. Longer waiting times may also delay diagnosis.

\section{Enriching the EuroQoL: does it work in ENT?}

T Kunanandam, G W McGarry, S Gatehouse, K MacKenzie From the ENT Department, North Glasgow Division, MRC Institute of Hearing Research, Glasgow, Scotland, UK

Aim

To assess the Enriched EuroQoL as a health status tool in ENT patients.

\section{Background}

The EuroQoL was developed to provide a standardised, generic, single index measure for describing and evaluating health-related quality of life. It can be applied at the individual, patient subgroup or population level.

This tool was developed by a multi-disciplinary group of researchers from seven centres across five different countries. It takes the form of a five-domain questionnaire which is suitable for self completion. The five domains are: mobility, self-care, usual activities, pain and discomfort, and anxiety and depression. Each domain has three levels, which in total identify 243 health states. Populationbased weightings can be applied to the raw data to derive a preference-based measure of health status, which is important for the justification of health resource allocation.

Although widely used, the EuroQoL appears to be deficient at detecting health-related quality of life issues in the ENT population. To address this ENT shortcoming, the Enriched EuroQoL has been devised. Enrichment adds a five-domain extension to the original questionnaire, the additional domains being communication, confidence and embarrassment, family activities, social activities and work, and effort and fatigue. The Medical Research Council is currently applying this new tool to patients with hearing aids, but as yet it has not been used in the wider ENT population.

Currently, there is no validated measure of health status in the ENT population.

We assessed the suitability of the Enriched EuroQoL as an appropriate tool for this purpose.

\section{Study design}

A prospective, controlled, population study.

Participants. Participants were identified from the specialist voice and rhinology clinics. Statistical review indicated that 150 complete datasets were required to reach significance.

Methods. Patients attending clinics at the Glasgow Royal Infirmary were asked to complete the Enriched EuroQoL questionnaire on arrival. Demographics and diagnosis were recorded for all participants. Complete datasets were entered onto an Excel spreadsheet and analysed using Statistical Package for the Social Sciences software. Values obtained for each of the 10 domains were compared with age- and sex-matched population controls.

\section{Results}

One hundred and sixty complete datasets were obtained. Seventy-two patients were included from the voice clinic and 88 from the rhinology clinic. Patients' male-to-female ratio was $1: 1.5$ and their age range was 19-88 years.

The results for domains one to five for the voice and rhinology patients did not indicate a significant difference from age- and sex-matched population controls.
However, the results for domains six to ten, i.e. the Enriched (ENT-specific) EuroQoL domains, did show a significant difference when compared with population controls.

\section{Conclusion}

This study indicates that the domains measured by the generic EuroQoL may not be appropriate for use in ENT. However, the Enriched EuroQoL seems to address this issue and may be more suited as a measure of health status in this patient group. We are continuing our work in this area and aim to validate the Enriched EuroQoL by comparing it with Time Trade-Off analysis, which is taken as the 'gold standard'.

\section{Patient satisfaction with the bone-anchored hearing aid: a 10-year experience}

A T M Mace, L D Cooke

From the North Glasgow Hospitals, Glasgow, Scotland, UK

\section{Objectives}

To evaluate the surgical problems, patient-rated quality of life and benefits associated with the use of bone-anchored hearing aids (BAHAs). Also, to identify potential improvements in comparison with previously used, conventional hearing aids.

\section{Study design}

Cross-sectional.

Setting

Bone-anchored hearing aid programme in North Glasgow, Scotland.

\section{Patients}

Fifty-nine adults who underwent BAHA implantation between 1995 and 2005.

\section{Main outcome measure}

Results of the Entific Medical Systems and Glasgow benefit inventory questionnaires.

\section{Results}

Thirty-four responses (58 per cent) were obtained. Patients' overall satisfaction was excellent $($ median $=10)$. The average total Glasgow benefit inventory score was 34. Bone-anchored hearing aid usage was high, with 85 per cent of patients wearing their BAHA for more than eight hours per day. Patients rated their BAHA as very good or excellent in single person conversation (91.2 per cent) and when listening to music, television or radio, but less satisfactory in group situations (44 per cent). Ear infections, where applicable, improved in 68 per cent of patients. Patients reported that their BAHA was better than their previous aid in terms of understanding language (82 per cent), sound comfort (82 per cent), cosmesis (68 per cent) and handling (68 per cent). The surgical complication rate related mostly to soft tissue problems (17 per cent). Revision surgery was required in 14 per cent of patients.

\section{Conclusions}

A BAHA can improve patient's quality of life. Improvements in hearing are achievable, in comparison with conventional hearing aids, but sound quality in group situations remains poor. Our results provide a predictive component to the pre-operative counselling of patients. 
Follow-up after ventilation tube insertion: what is needed and when? A completed audit loop

H McKee, P M Spielmann, R M Adamson, G Thiel, D Schenk, S S M Hussain

From the Department of Otolaryngology, Ninewells Hospital and Medical School, Dundee, Scotland, UK

\section{Background}

There is a paucity of evidence to guide the post-operative follow up of patients undergoing their first ventilation tube insertion. Two loops of data collection and analysis were performed. All paediatric patients undergoing ventilation tube insertion for the first time were identified. Patients who had had a previous ventilation tube insertion or additional procedures such as adenoidectomy or tonsillectomy were excluded. The findings of the first study prompted a change in the post-operative regime. All patients undergoing ventilation tube insertion were subsequently seen at three months and a pure tone audiogram taken. Further review depended on the clinical and audiometric findings.

\section{Results}

First cohort. Fifty patients met the inclusion criteria. There were a total of 156 appointments between surgery and data collection. A total of 131 (84 per cent) appointments lead to no medical intervention. The only statistically significant difference between the group requiring further ventilation tube insertion $(n=10)$ and those not requiring further treatment during the study period $(n=40)$ was the average hearing threshold $(p<0.01)$.

Second cohort. Records for 84 children were collected, representing a total of 154 appointments. In only 18 appointments (12 per cent) were normal findings and hearing recorded and children given a further review appointment. Sixteen of the 29 (55 per cent) children with abnormal clinical findings (i.e. otorrhoea, tube blockage or extrusion) required some form of intervention. This was statistically significant $(p<0.05)$. Twenty-six children had a mean hearing threshold worse than $20 \mathrm{~dB}$ at first review. Nineteen (73 per cent) required further intervention of some sort; this was also statistically significant ( $p$ $<0.01)$.

\section{Discussion}

Our study demonstrates that the vast majority of review appointments resulted in no clinical intervention. We therefore question the need for regular follow up in this patient group. Twenty per cent (10 of 50 and 18 of 84 ) of our patients required further grommet insertion within the study periods. This is consistent with reported rates in the literature. A child with abnormal clinical findings or a mean hearing threshold of less than $20 \mathrm{~dB}$ was significantly more likely to require further intervention.

We would recommend one post-operative review, with audiometry, at three months after surgery. At this initial appointment, further review should be offered to those children with poor hearing, early extrusion or infection, as they are more likely to require further grommet insertion.

\section{Evaluation of functional endoscopic sinus surgery by Glasgow benefit inventory}

J R Newton, M Shakeel, B Ram

From the Aberdeen Royal Infirmary, Aberdeen, Scotland, UK

Functional endoscopic sinus surgery (ESS) is a common adjunct to medical therapy in cases of chronic rhinosinusitis and nasal polyposis.
The objective of this study was to assess patients' quality of life up to two years after ESS.

We analysed 50 consecutive patients attending a rhinology clinic who fitted our criteria, and assessed their subjective rating of benefit using the Glasgow benefit inventory, a validated questionnaire. The patients were divided into three groups according to the time period elapsed since surgery (i.e. six months, 12 months and up to two years). The Glasgow benefit inventory post-interventional survey incorporates general, social support and physical health subscales and provides detailed information regarding the nature of benefit experienced after surgery.

The resulting scores generally indicated a benefit from the procedure. Overall, surgery led to statistically significant improvements in both total, general and physical scores $(p<0.05)$. Comparison of ESS with nasal polypectomy (plus ESS) indicated a greater benefit for polyp disease in the total score and in the general subscale $(p=0.031, p=0.029)$. No statistical difference was observed when comparing the scores for females $v s$ males or for different follow-up periods.

Overall, this study supports the effectiveness of ESS in producing prolonged improvement in patients' quality of life, in addition to symptomatic benefits.

\section{Long-term effectiveness of antiseptic cream for recurrent epistaxis in childhood: five-year follow up of a randomised, controlled trial}

S Robertson, H Kubba

From the Department of Paediatric Otolaryngology, Royal Hospital for Sick Children, Glasgow, Scotland, UK

\section{Introduction}

In 2001, a randomised, controlled trial demonstrated that children given antiseptic cream for four weeks prior to their first clinic visit were significantly more likely to have complete resolution of epistaxis than children receiving no treatment. However, the long-term effectiveness of any treatment, including antiseptic cream, is unknown.

\section{Aims and methods}

Our aim was to determine the long-term outcome of treatment for recurrent epistaxis in children. Five-year follow-up data were collected from the randomised, controlled trial. Ethical committee approval was obtained. Data were collected by telephone interview, case record review and from the hospital's computer system.

\section{Results}

Case note and computer record review achieved follow up for 78 of 88 children ( 89 per cent). At their first clinic visit in 2001, 24 children received further cream (eight from the treatment group and 16 controls), 44 underwent cautery and received cream (23 from the treatment group and 21 controls), and 10 received no treatment. Since 2001, 19 (22 per cent) had been referred back to the clinic. Only five children had attended the emergency room, and eight had required cautery.

In total, 60 of 88 children were contactable by telephone. Twenty-three of 36 (64 per cent) from the original naseptin group and 16 of 24 (67 per cent) from the original control group were still having ongoing bleeding $(p=0.82)$. Eighteen of the 39 children with ongoing bleeding (46 per cent) were bleeding at least monthly. Only 11 children (18 per cent) had used further cream since 2001.

\section{Conclusion}

The majority of children with recurrent epistaxis referred to our clinic had resolution of bleeding in the short-term 
with treatment, yet many appeared to have suffered ongoing bleeding in the long-term. Few children attended the emergency room and few received further antiseptic cream.

\section{Use of complementary and alternative medicine by patients attending a general otolaryngology clinic}

M Shakeel, S A Little, A Evans, E Liu, K W Ah-See

From the Department of Otolaryngology, Aberdeen Royal Infirmary, Aberdeen, Scotland, UK

\section{Background}

There is growing interest in and use of complementary and alternative medicine amongst the general population. However, little information is available on the use of such medicine by adults attending UK otolaryngology out-patient clinics.

\section{Objective}

To study the prevalence and pattern of complementary and alternative medicine use among adult patients attending the ENT clinic in a UK teaching hospital.

\section{Patients and method}

All patients on their primary visit to the ENT clinic were asked to complete an anonymous questionnaire over a 14-week period from October 2005 to January 2006.

Results

Based on 1366 completed questionnaires, 53 per cent (728/ 1366) of respondents were female and 47 per cent $(638 /$ 1366) were male. Twenty-nine per cent $(395 / 1366)$ were older than 60 years and 56 per cent $(763 / 1366)$ were married. Twenty-six per cent $(357 / 1366)$ had a university qualification. Sixty-one per cent $(833 / 1366)$ had used complementary and alternative medicine, 36 per cent in the preceding 12 months. The popular remedies were cod liver oil (368/833), garlic $(197 / 833)$, cranberry $(181 / 833)$, aloe vera $(176 / 833)$, primrose oil $(174 / 833)$ and echinacea $(163 / 833)$. Non-herbal therapies included massage (230/ $833)$, acupuncture (186/833), aromatherapy (135/833), chiropractic (121/833), reflexology (119/833) and homeopathy (110/833). Seventeen per cent (143/833) used complementary and alternative medicine for their current illness. Only 8 per cent $(64 / 833)$ found complementary and alternative medicine ineffective, yet only 57 per cent (473/833) would recommend complementary and alternative medicine to others. Fifty-one per cent (421/833) failed to inform their primary physician of their complementary and alternative medicine use.

\section{Discussion}

Despite concerns over the efficacy, safety and costeffectiveness of complementary and alternative medicine, use of such medicine was popular among patients attending our ENT clinic. Use of such medicine is not generally related to patients' presenting illness. Otolaryngologists should be aware of current trends in complementary and alternative medicine use when managing patients, including possible interactions with other medication.

\section{Open-ended questionnaires and the dizzy patient}

\section{E Stapleton, R Mills}

From the Otolaryngology Unit, University of Edinburgh, Edinburgh, Scotland, UK

A clear and detailed clinical history is essential in the assessment of patients with balance symptoms. The aim of this study was to assess the usefulness of open-ended questionnaires in the specialist balance clinic.

Fifty-four consecutive new patients completed an openended questionnaire prior to a consultation in which the history was taken using a standardised proforma. The results of the two were compared.

Open-ended questionnaires provide patient-centred data and do not provide clinicians with sufficient data for diagnosis. Patients are more likely to respond in the affirmative when asked about symptoms directly, than to report the same symptoms spontaneously on an open-ended questionnaire. When questions have a number of possible answers, patients are more likely to report them in full in an openended questionnaire than to provide a response during direct questioning.

A clear and detailed clinical history can be exceptionally difficult to ascertain in patients with balance symptoms. We found that patients could give contradictory (and hence unreliable) information when their history was taken using different methods. This highlights the need for meticulous attention to accuracy and detail when the clinician is dealing with a dizzy patient.

\section{Correlation of radiological findings and clinical outcome in pharyngeal pouch stapling}

A Tsikoudas, D Eason, $\mathrm{N}$ Kara, J N Brunton, R E Mountain

From the Departments of Otolaryngology and Radiology, Ninewells Hospital, Dundee, Scotland, UK

\section{Objective}

We undertook to identify any correlation between radiological findings and clinical outcome in pharyngeal pouch surgery.

\section{Methods}

We performed a retrospective case-control study within a teaching hospital in Dundee, Scotland. The subjects were 21 patients who had undergone pharyngeal pouch stapling. We used a novel method to measure the characteristics of each pouch by focussing on a triangular area in the neck of the pouch.

Results

A correlation was found between the pre-operative radiological findings and the clinical outcome. A short triangle was more likely to result in post-operative complications, while a long one was more likely to require revision surgery.

\section{Conclusions}

It is possible to predict the surgical outcome from the radiological characteristics of the diverticulum. We believe that our findings can have important clinical implications.

(Note that this paper has previously been published in the October issue of the US journal Annals of Otology, Rhinology \& Laryngology.)

\section{Microbiology of childhood epistaxis}

A Whymark, L Fraser, D Crampsey, S Ali, P Moore, C Williams, H Kubba

From the ENT Department, Royal Hospital for sick children yorkhill, Glasgow

\section{Introduction}

We have previously shown that antiseptic cream is an effective treatment for childhood epistaxis, ${ }^{1}$ while petroleum 
jelly is ineffective. ${ }^{2}$ This implies that epistaxis in childhood has a microbacterial aetiology. We hypothesise that childhood epistaxis results from nasal colonisation with Staphylococcus aureus, leading to the characteristic inflammation, crusting and new vessel formation identified in previous pathological studies. ${ }^{3}$

\section{Methods}

A prospective case-control study was undertaken. Research ethics committee approval was obtained. Children referred to our ENT clinic with epistaxis were categorised as having crusting or no crusting following nasal examination. A microbiology swab was taken from the affected nasal vestibule and sent for culture and sensitivity. Control subjects without epistaxis were recruited from the orthopaedic and accident and emergency clinics, and a similar nasal microbiology swab was taken.

\section{Results}

Forty-two patients with epistaxis were recruited. Of these, 22 had crusts and 20 did not. Twenty-five control subjects were also recruited. The age and sex ratios of each group were statistically similar. Analysis of the microbiology results revealed that all groups were equally likely to have a positive culture (crusts $14 / 22$, no crusts $13 / 20$, controls $\left.14 / 25 ; x^{2}=0.46, \mathrm{df}=2, p=0.79\right)$. Staphylococcus aureus was more common in the groups with epistaxis, compared with control subjects (crusts $12 / 22$, no crusts $12 / 20$, controls $6 / 25 ; x^{2}=7.09, \mathrm{df}=2, p=0.03$ ). There was no difference in the prevalence of Staph aureus between crust and non-crust groups. The majority of the other control subjects either had no bacteria isolated $(11 / 25)$ or had isolates of respiratory pathogens $(4 / 25)$ or commensals $(4 / 25)$. Epistaxis patients were much less likely to have isolates of respiratory pathogens (1/22 crusts, $1 / 20$ crusts) or commensals (0/22 crusts, $0 / 20$ no crusts).

\section{Conclusion}

Regardless of whether or not there is crusting, children with epistaxis were much more likely to have nasal colonisation with Staph aureus than were controls. Our data support the hypothesis that Staph aureus replaces existing nasal flora, and we believe that this is the cause of the inflammation and new vessel formation seen in previous pathological studies.

\section{References}

1 Kubba H, MacAndie C, Botma M, Robison J, O'Donnell M, Robertson $\mathrm{G}$ et al. A prospective single-blind randomised controlled trial of antiseptic cream for recurrent epistaxis in childhood. Clin Otolaryngol 2001;26:465-8

2 Loughran S, Spinou E, Clement WA, Cathcart R, Kubba H, Geddes NK. A prospective, single-blind, randomised controlled trial of petroleum jelly (Vaseline) for recurrent paediatric epistaxis. Clin Otolaryngol 2004;29:266-9

3 Montague ML, Kubba H. Pathology of epistaxis.

\section{Incidence of thyroid gland invasion by laryngeal carcinoma}

\section{Wynne}

From the ENT department, North Glasgow Hospitals, Glasgow, Scotland, UK

\section{Objective}

To audit the incidence of thyroid gland invasion by laryngeal carcinoma.

\section{Study design}

Retrospective audit of laryngectomy specimens.
TABLE I

AUDIT OF PREVIOUS LARYNGECTOMY SPECIMENS*

\begin{tabular}{lrrccc}
\hline Invasion? & Supra & Glottic & Sub & Trans & $\begin{array}{c}\text { Pyriform } \\
\text { sinus }\end{array}$ \\
\hline No & 12 & 13 & 0 & 7 & 7 \\
Yes & 0 & 0 & 2 & 3 & 0 \\
\hline
\end{tabular}

Total $n=44$. Supra $=$ supraglottic; sub $=$ subglottic; trans $=$ transglottic

\section{Setting}

Otorhinolaryngology department within the North Glasgow University NHS trust.

\section{Inclusion criteria}

Laryngectomy specimen.

\section{Discussion}

Traditionally, laryngectomy for squamous carcinoma has been performed in conjunction with ipsilateral hemithyroidectomy. Within the published literature, a few papers have questioned whether this is required routinely in all tumour subgroups. Reported results include the following: 0 per cent invasion in glottic/ supraglottic carcinoma (1995, Hong Kong); 1 per cent invasion in transglottic carcinoma (1995, Hong Kong); 1 per cent invasion in transglottic carcinoma (2001, Turkey); and 8 per cent invasion in all stage IV disease (1991, Denver).

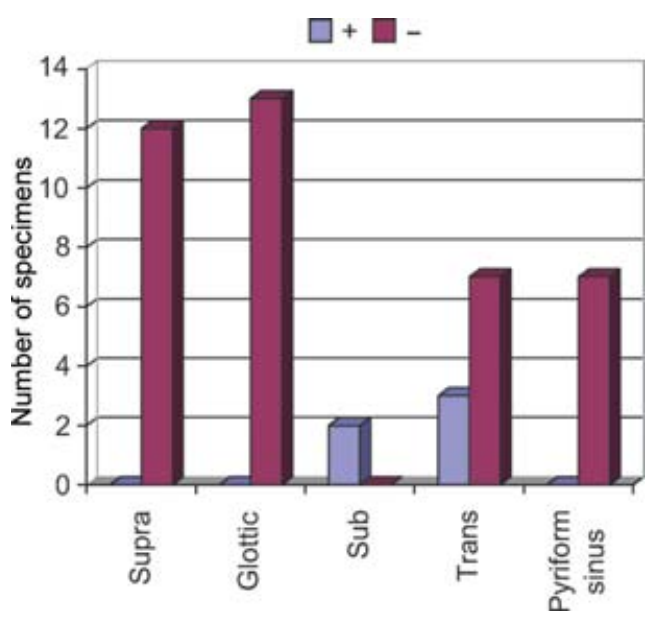

FIG. 1

Incidence of thyroid gland invasion Supra = supraglottic; sub $=$ subglottic; trans $=$ transglottic 
However, following searches of the Medline, OVID and Pubmed databases, no published UK data on this issue could be found.

A retrospective audit of previous laryngectomies performed in the North Glasgow NHS trust was recently undertaken (see Table I and Figures 1 and 2). This showed that thyroid invasion occurred only in patients with subglottic involvement or those requiring emergency tracheostomy prior to laryngectomy.

Ten patients had no comment on thyroid gland invasion but the tumour had not extended to the thyroid cartilage. Two patients required tracheostomy; both had thyroid invasion. 